\title{
An adaptive controller based upon continuous estimation of the closed loop frequency response
}

\author{
JENS G. BALCHEN and BERNT LIE†
}

Keywords: Adaptive control; frequency response; correlation methods; parameter estimation; process control.

\begin{abstract}
An adaptive control algorithm based upon on-line estimation of the -180 degree shift frequency and the gain margin is investigated. The estimates are found by perturbing the system with a small signal and then correlating the system deviation with the excitation. As only two parameters are estimated, the algorithm is robust. Moreover, the estimator can be applied to non-minimum phase systems, including systems with a varying time-delay. The control algorithm can be of any form as long as there is a functional relationship between the estimated parameters and the control algorithm parameters. To avoid an excessive perturbation signal, the square of the amplitude of this signal is automatically adjusted to a certain ratio of the variance of the error signal. A mathematical analysis of convergence is presented and the theory is confirmed by experimental results.
\end{abstract}

\section{Introduction}

In the design of control systems for monovariable processes, a number of the process properties are the most important determinants of the behaviour of the total system. As is well known from classical control theory, the open loop frequency response behaviour in the neighbourhood of frequency $\omega_{180}$ (the frequency at which the phase shift of the loop transfer function is -180 degrees) has a very strong influence on the closed loop control performance.

A number of different types of models can be used to express the behaviour of the process with reference to control. State space models express most details about the internal behaviour of the process whereas input-output models offer compact parametric descriptions for linear systems.

Adaptive or self-tuning controllers for monovariable processes are usually based upon discrete transfer function models, e.g.

$$
h_{p}\left(z^{-1}\right)=\frac{b_{1} z^{-1}+b_{2} z^{-2}}{1+a_{1} z^{-1}+a_{2} z^{-2}} z^{-m}
$$

where the polynominal coefficients in the model are estimated using efficient on-line algorithms (Ljung and Söderstrom, 1985). Depending on the control strategy, the coefficients of the discrete controller transfer function are given by an explicit formula of the estimated parameters of the process model ( $\AA$ ström et al., 1977).

Received 15 June 1987

$\dagger$ Division of Engineering Cybernetics, The Norwegian Institute of Technology, 7034 Trondheim, Norway.

This paper was presented at the IFAC Workshop on Adaptive Systems in Control and SignalProcessing, Lund, Sweden, 1-3 July 1986, and is reprinted with the permission of IFAC. 
Though this approach to the design of adaptive or self-tuning controllers has many advantages, there are also some drawbacks. Some of the disadvantages are:

- The adaptive control algorithm in its ordinary form does not contain a provision for assuring proper excitation of the process which will make the parameter estimator perform satisfactorily. If the process excitations are too small or have improper frequency distributions, the variance of the parameter estimates may become significant resulting in low performance control. In the concept of dual control (Feldbaum, 1960) the generation of proper process excitations which facilitate efficient parameter estimation appears as an integral part of the algorithm. 'Dual control' is, however, rarely implemented in existing adaptive control schemes.

- The type of parameterization of the process model used in (1.1) is efficient for the implementation of time-discrete controllers, but does not efficiently reflect the most significant properties of the control loop. Thus much of the computational burden on the adaptive controller may be irrelevant.

- The ability of an adaptive controller to cope with changing amounts of transportation lag in the process dynamics is not always satisfactory. In fact, most adapative algorithms are based on the assumption that the transportation lag is fixed and known. In many industrial processes, the transportation lag is very much dependent upon process conditions and should be taken into account in the adaptation procedure.

In this paper an approach to adaptive control is suggested which is based upon a description of the control loop in the frequency domain. The two most significant parameters in the frequency response $\left(h_{0}(j \omega)\right)$ of the control loop are estimated on-line and are used to directly adjust the controller parameters. Since there are only two parameters to estimate, the algorithm is robust. The parameters, which are estimated, will automatically handle non-minimum phase systems, e.g. systems with varying time delays. The robustness is further improved by including an algorithm which assures proper excitation of the process.

\section{Basic principles of the new adaptive controller}

In Fig. 2.1, a block diagram indicates the functions of the adaptive control system. At the setpoint input to the controller, a small sinusoidal perturbation signal $(\delta y)$ is added and the response in the system deviation is fed to the

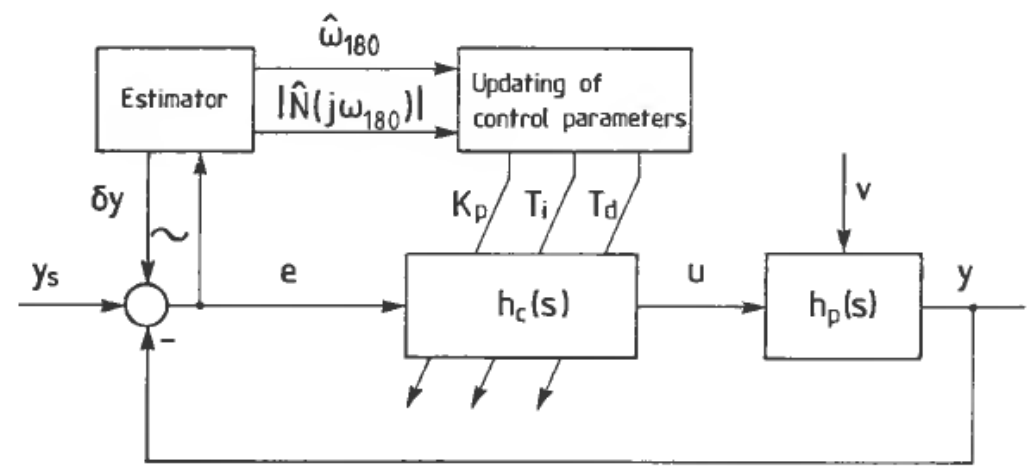

Figure 2.1. Basic scheme of the adaptive controller. 
'Estimator'. This unit adjusts the frequency of the perturbation signal so that it reaches $\omega_{180}$ (the frequency at which the open loop frequency response $h_{0}(j \omega)$ has a phaseshift of $\left.\angle h_{0}(j \omega)=-180^{\circ}\right)$. The estimated value of this frequency is referred to as $\hat{\omega}_{180}$. The Estimator also determines the magnitude of the "disturbance reduction ratio'

$$
N(j \omega)=\frac{1}{1+h_{0}(j \omega)}
$$

at the particular frequency, and this estimated value is denoted by $\left|\hat{N}\left(j \omega_{180}\right)\right|$. Figure 2.1 also indicates how typical parameters of a PID controller (proportional gain $K_{p}$, integral time $T_{i}$, derivative time $T_{d}$ ) are adjusted based upon the estimated system parameters.

Figure 2.2 shows the frequency response characteristics for three types of systems. One major difference between these plots, is the number of integrations encountered in the systems. System nos. 1, 2 and 3 have zero, one and two integrations, respectively. The frequency $\omega_{180}$ is easily recognized in Fig. 2.2, and the contours of constant $|N(j \omega)|$ are given by the inverted Nichols chart. The gain margin $\Delta K$ of the control loop is recognized as the distance along the logarithmic gain scale between point $\omega_{180}$ and point $\left(\left|h_{0}\right|=0 \mathrm{~dB}, \angle h_{0}=-180^{\circ}\right)$. Consequently, we may find a simple relationship between $N\left(j \omega_{180}\right)$ and $\Delta K$. Since $h_{0}\left(j \omega_{180}\right)=-1 / \Delta K$ we have

$$
N\left(j \omega_{180}\right)=\frac{1}{1-\frac{1}{\Delta K}}=\frac{\Delta K}{\Delta K-1}
$$

A method which is frequently used for the adjustment of controller parameters is specifying a certain gain margin and a phase margin for the control loop. In most cases of process control, the gain margin will be the determining factor because most processes encompass some transportation lag or another type of nonminimum phase behaviour which results in a rapidly increasing negative phase shift with increasing frequency. If we want the control loop to have a specified gain

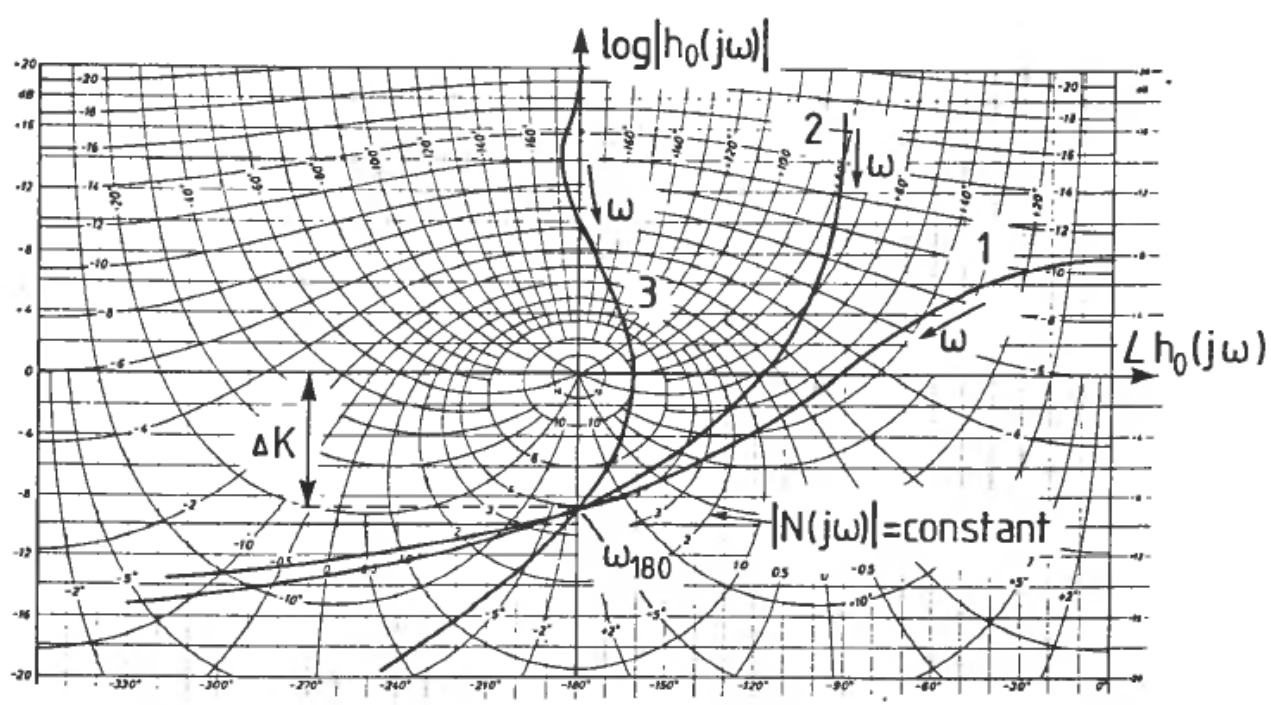

Figure 2.2. Frequency response characteristics for three types of systems. 
margin of $\Delta K$, it follows that we have a specified value of $N\left(j \omega_{180}\right)$ according to 2.2 . Furthermore it is easily verified that for a stable system, $\angle N\left(j \omega_{180}\right)=0^{\circ}$ so that $N\left(j \omega_{180}\right)=\left|N\left(j \omega_{180}\right)\right|$.

By adjusting the proportional gain of the controller in a feedback sense until $\left|N\left(j \omega_{180}\right)\right|$ reaches its specified value, the first part of the controller adaptation has been achieved.

The adaptation of the dynamic parameters of the controller, i.e., integral time $T_{i}$ and derivative time $T_{d}$ in a PID controller, is conveniently done by relating these factors directly to $\omega_{180}$. In the simplest case the following relationships are chosen:

$$
\begin{aligned}
& \frac{1}{T_{d}}=\gamma \omega_{180} \quad \text { with } \quad \gamma \approx 0 \cdot 5-1 \cdot 0 \\
& \frac{1}{T_{i}}=\delta \frac{1}{T_{d}} \quad \text { with } \quad \delta \approx 0 \cdot 1-0 \cdot 4
\end{aligned}
$$

It is thus up to the designer to choose the parameters $\gamma$ and $\delta$. In most cases of normal industrial control, this choice will not be very critical, but it is necessary that the designer has a basic understanding of the mechanisms of the process so that he can make a reasonably valid choice. The same type of relationships are well known from classical tuning techniques such as the Ziegler-Nichols method for instance.

The basic pricinciple of the adaptive controller to be described, is thus as follows:

(1) Frequency $\omega_{180}$ for the closed loop and quantity $\left|N\left(j \omega_{180}\right)\right|$ are estimated using a continuous experimental technique.

(2) The controller gain is adjusted until a prescribed value of $\left|N\left(j \omega_{180}\right)\right|$ is reached, thereby achieving a certain gain margin $(\Delta K)$ in the loop.

(3) Dynamic parameters of the controller such as integral time $\left(T_{i}\right)$ and derivative time $\left(T_{d}\right)$ are adjusted in a fixed relationship to $\omega_{180}$.

\section{The Estimator}

\subsection{Estimation of $\omega_{180}$}

Figure 3.1 shows a block diagram of the basic system for estimating $\omega_{\mathbf{1 8 0}}$. It consists of a signal generator which produces a sine signal and a cosine signal with adjustable frequency. The sine signal also has adjustable amplitude $(\Delta y)$. The small sinusoidal perturbation $(\delta y)$ is added to the regular setpoint $\left(y_{s}\right)$ thereby producing the total controller setpoint $\left(y_{0}\right)$. The system deviation is denoted $e$ and the total amount of disturbances acting upon the loop which is referred to the output of the process is denoted $v$. The Laplace transform of these signals yield the relationship

$$
e(s)=N(s) y_{0}(s)-N(s) v(s)
$$

In Fig. 3.1 the frequency of the signal generator may be changed. In the simplified analysis which follows, it is assumed that the rate of change in this frequency is rather low. It is of course also assumed that due to the small perturbations, a linearized description such as that of (3.1) is valid. 


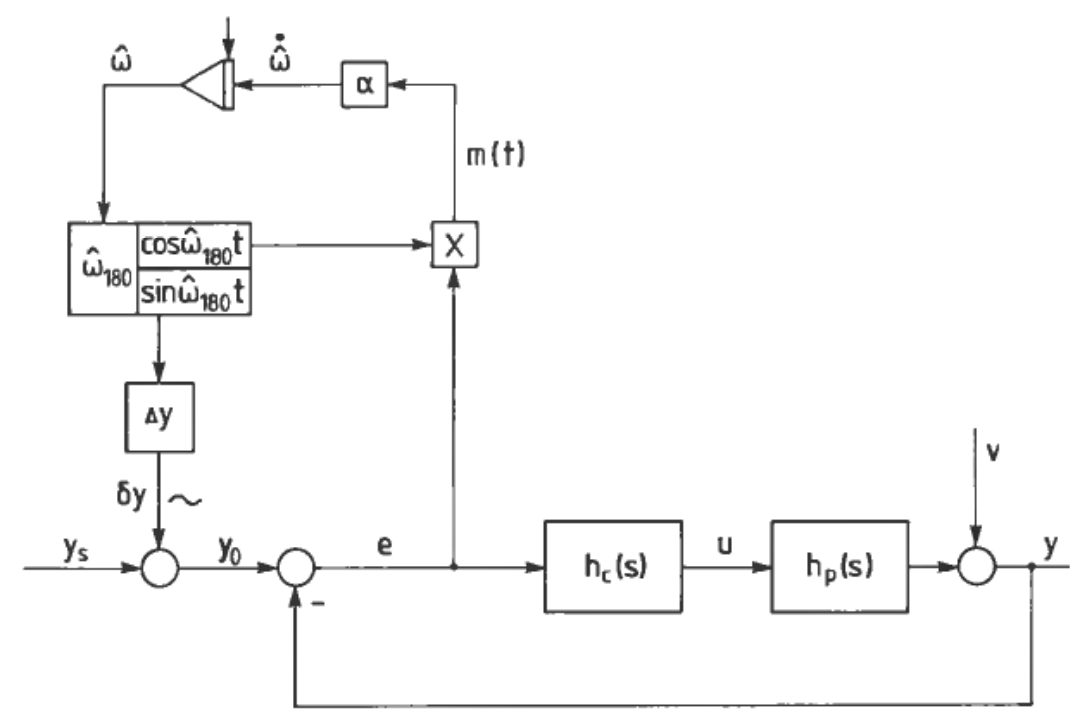

Figure 3.1. Basic scheme for estimation of $\omega_{180}$.

If it is assumed that the closed loop contains an integral action and that the setpoint $\left(y_{s}\right)$ is constant, the time response of the system deviation will be

$$
e(t)=\Delta y\left|N\left(j \hat{\omega}_{180}\right)\right| \sin \left[\hat{\omega}_{180} t+\angle N\left(j \hat{\omega}_{180}\right)\right]-\tilde{v}(t)
$$

in which $\tilde{v}(t)$ is the time domain representation of the last term in (3.1). The frequency of perturbation is denoted $\hat{\omega}_{180}$. This is going to be the estimated value of $\omega_{180}$.

As is seen from Fig. 3.1, the rate of change in the perturbation frequency $\left(\dot{\hat{\omega}}_{180}\right)$ is made proportional to the product of the response in the system deviation $(e(t))$ and $\cos \hat{\omega}_{180} t$. This is so because at $\omega_{180}$ the phase shift of the sinusoidal response will be $\angle N\left(j \omega_{180}\right)=0^{\circ}$. Since $\sin \omega t$ and $\cos \omega t$ are orthogonal functions, the signal produced by the multiplier will on average be equal to zero at $\omega_{180}$. The factor $\alpha$ in Fig. 3.1 constitutes the gain of the estimation loop and determines the rate of convergence. The output of the multiplier in Fig. 3.1, $m(t)$ will become

$$
\begin{gathered}
m(t) \approx \frac{1}{2} \Delta y|N|\left[\sin \left(2 \hat{\omega}_{180} t+\angle N\right)+\sin \angle N\right] \\
-\tilde{v}(t) \cos \hat{\omega}_{180} t
\end{gathered}
$$

in which the simplified notation

$$
|N|=\left|N\left(j \hat{\omega}_{180}\right)\right| \quad \text { and } \quad \angle N=\angle N\left(j \hat{\omega}_{180}\right)
$$

has been introduced. Normally the process disturbances will have a stochastic nature and since the closed loop is assumed to have integral action, the average value of $\tilde{v}(t)$, and thus the average value of the last term in (3.3), will be zero. Furthermore it is observed that the first term in the bracket of (3.3) is sinusoidal with the frequency $2 \hat{\omega}_{180}$. This means that there will be a small modulation of the frequency around an average. The frequency of this modulation will be $2 \hat{\omega}_{180}$ as indicated in Fig. 3.2. Neglecting this modulation which will be small when $\alpha$ is small, the approximate differential equation for the estimator will be given

$$
\dot{\omega}_{180}=\frac{1}{2} \alpha \Delta y|N| \sin \angle N
$$




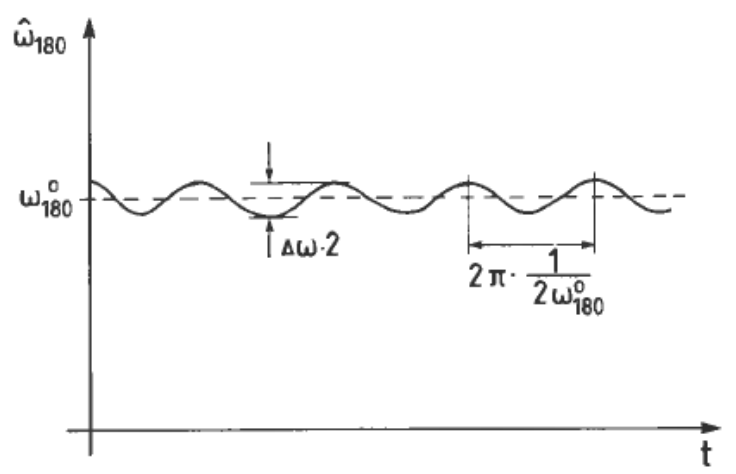

Figure 3.2. The oscillation in the estimate of $\omega_{180}$.

The stability of the $\omega_{180}$ estimation loop depends on how $\sin \angle N$ varies with $\omega$. Figure 3.3 shows some typical forms of $\sin \angle N(j \omega)$ for two of the systems shown in Fig. 2.2, where system type 2 is regarded to be the most common in process control. This system is seen to have a 'domain of attraction' which is $0<\hat{\omega}_{180}<\omega_{H}$ in which $\omega_{H}$ is the next frequency above $\omega_{180}$ where $\angle N(j \omega)=0^{\circ}$. In the case of a system of type 3, it is seen from Fig. 3.3(b) that the 'domain of attraction' is determined by $\omega_{L}<\hat{\omega}_{180}<\omega_{\mathrm{H}}$ in which $\omega_{L}$ is the frequency below $\omega_{180}$ at which $\angle N(j \omega)=+180^{\circ}$.

The consequence of the above discussion is that for a system of type 2, the convergence of the estimation loop is secured if the estimator is started at a sufficiently low frequency, whereas the case of a system of type 3 , one must simultaneously ensure that the system is started at a frequency above $\omega_{L}$.

The slight oscillation of the frequency depicted in Fig. 3.2 can be roughly estimated by assuming that the steady state solution of the actual system is

$$
\hat{\omega}_{180}(t)=\omega_{180}^{\circ}+\Delta \omega \sin \left(2 \omega_{180}^{\circ} t+\varphi\right)
$$

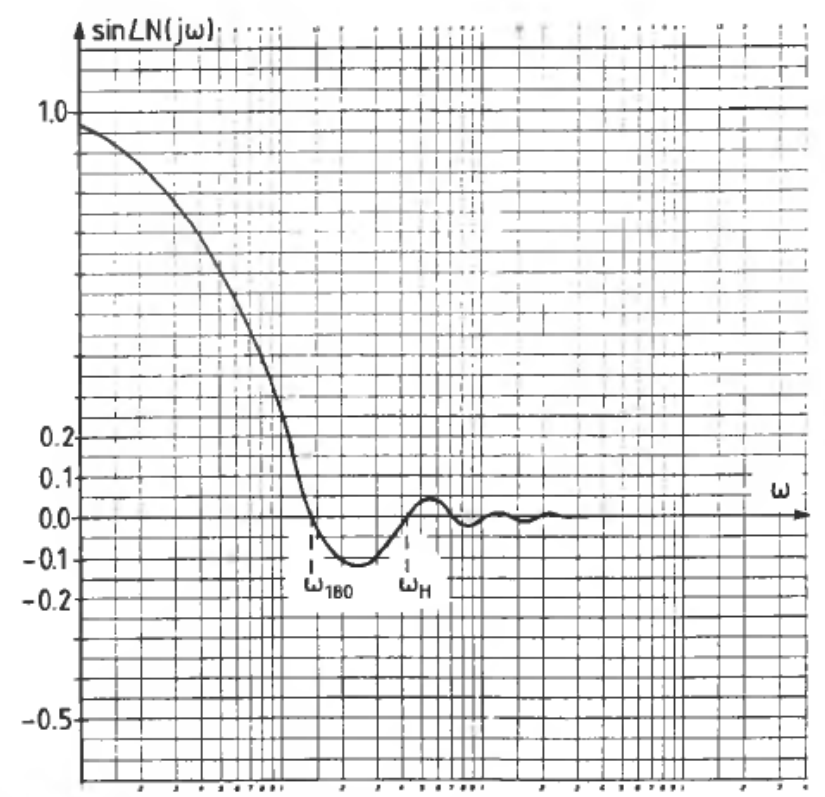

Figure 3.3(a). Domain of attraction for systems with frequency response characteristics such as type no. 2 in Fig. 2.2. 


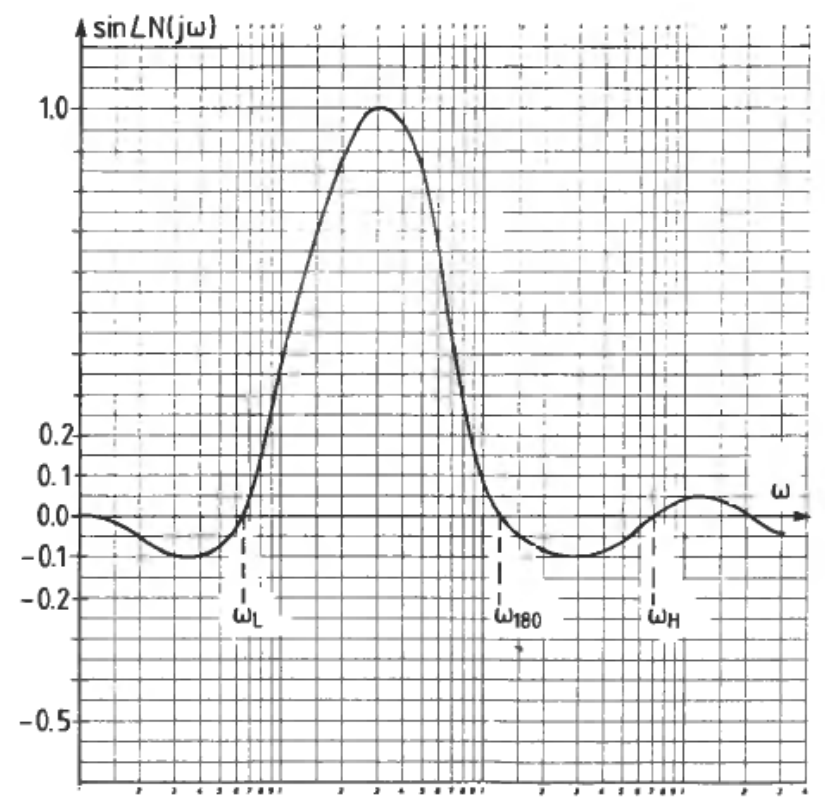

Figure 3.3(b). Domain of attraction for systems with frequency response characteristics such as type no. 3 in Fig. 2.2 .

It can be shown that

$$
\Delta \omega \approx \frac{\alpha \Delta y}{4 \omega_{180}^{\circ}}\left|N\left(j \omega_{180}^{\circ}\right)\right|
$$

\subsection{Estimation of $\left|N\left(j \omega_{180}\right)\right|$}

In Fig. 3.4 a block diagram is shown of the part of the estimator which deals with the quantity $\left|N\left(j \omega_{180}\right)\right|$. The difference between the real sinusoidal deviation signal and the estimated deviation (assuming integral action in the closed loop) is

$$
\delta e(t)=e(t)-\hat{e}(t) \approx \Delta y|N| \sin \left(\hat{\omega}_{180} t+\angle N\right)-\tilde{v}(t)-\Delta y|\hat{N}| \sin \left(\hat{\omega}_{180} t\right)
$$

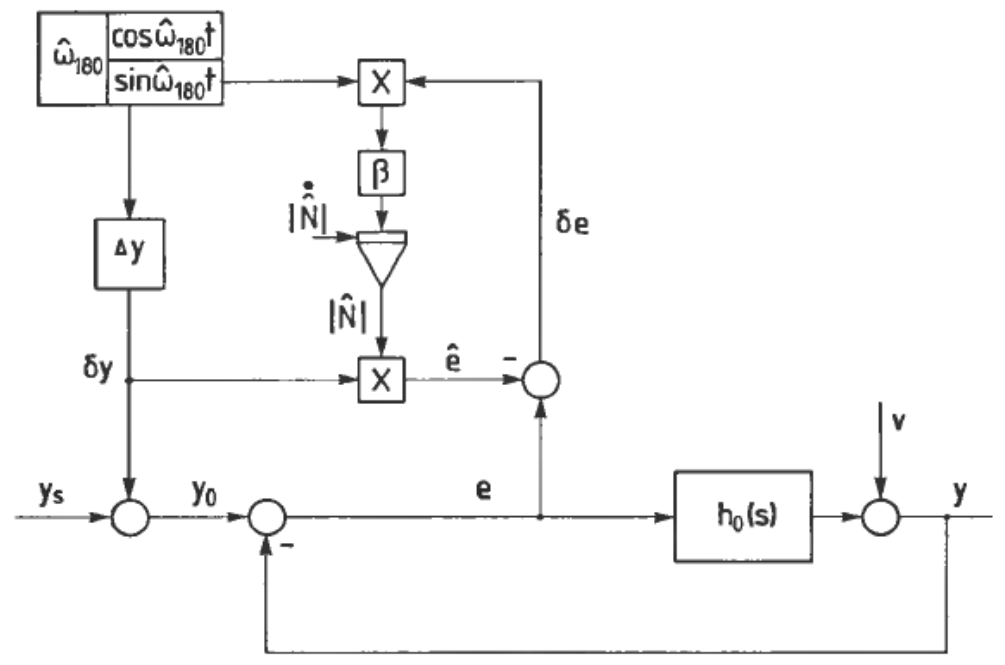

Figure 3.4. Basic scheme for estimation of $\left|N\left(j \omega_{180}\right)\right|$. 
Here $|\hat{N}|$ is a scalar quantity generated by an integrator, this scalar being the previously mentioned estimate of $\left|N\left(j \omega_{180}\right)\right|$. If it is assumed that factor $\beta$ in Fig. 3.4 has a small numerical value, the rate of change $|\dot{\hat{N}}|$ in the output of the integrator will be small. The system in Fig. 3.4 tends to make the cross-correlation equal to zero between the difference signal $\delta e$, which is a sinusoide, and the sinusoidal perturbation signal. This is seen by formulating the differential equation governing the system in Fig. 3.4.

$$
|\hat{\hat{N}}|=\beta \sin \left(\hat{\omega}_{180} t\right) \delta e(t)
$$

Applying (3.7) into (3.8) and neglecting the influence of the stochastic disturbance, we get

$$
|\hat{N}| \approx \beta \frac{\Delta y}{2}\left[|N|\left(\cos \angle \mathrm{N}-\cos \left(2 \hat{\omega}_{180} t+\angle N\right)\right)-|\hat{N}|\left(1-\cos 2 \hat{\omega}_{180} t\right)\right]
$$

Equation (3.9) shows that the input to the integrator contains a sinusoidal oscillation with the frequency $2 \hat{\omega}_{180}$. Assuming now that the loop for estimating $\omega_{180}$ has converged and therefore $\angle N \approx 0^{\circ}$, and by taking the average of (3.9) over some periods of the frequency $2 \hat{\omega}_{180}$, the differential equation (3.9) will approach the following:

$$
|\dot{\hat{N}}| \approx \beta \frac{\Delta y}{2}(|N|-|\hat{N}|)
$$

This is a stable differential equation where $|\hat{N}|$ approaches $|N|$ with a time constant

$$
T_{|N|}=\frac{2}{\beta \Delta y}
$$

\section{Adaptation of the controller}

\subsection{Adjustment of the controller parameters}

Any control algorithm may be adapted on the basis of the estimates $\hat{\omega}_{180}$ and $\left|\hat{N}\left(j \omega_{180}\right)\right|$. Here we shall look at the adaptation of a PID controller with the transfer function

$$
h_{c}(s)=K_{p} \frac{\left(1+T_{i} s\right)\left(1+T_{d} s\right)}{T_{i} s}
$$

It is proposed that the adaptation of this controller is done according to the block diagram in Fig. 4.1. Here it is seen that the estimate $|\hat{N}|$ is compared to a desired value $|N|_{0}$ and an integral controller is used to adjust the proportional gain $K_{p}$ of the main controller of (4.1). Furthermore the integral time $T_{i}$ and the derivative time $T_{d}$ are adjusted according to the simple relationships given previously

$$
\begin{aligned}
& \frac{1}{T_{d}}=\gamma\left|\hat{\omega}_{180}\right| \\
& \frac{1}{T_{i}}=\delta \frac{1}{T_{d}}=\delta \gamma\left|\hat{\omega}_{180}\right|
\end{aligned}
$$




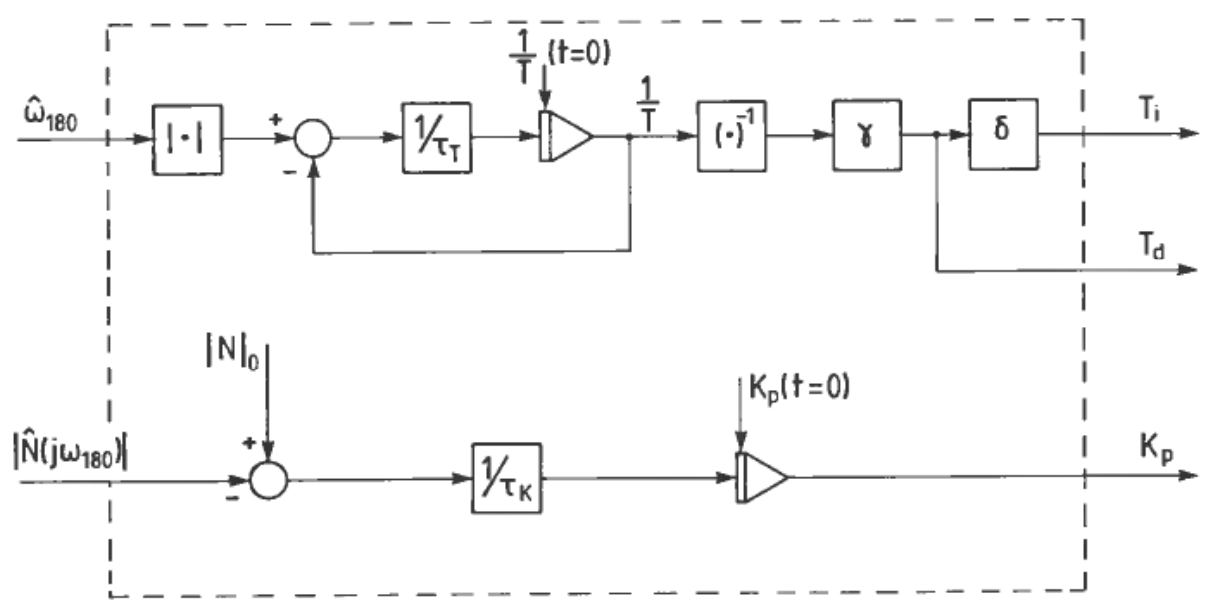

Figure 4.1. Scheme for adapting the control parameters.

The reason for using $\left|\hat{\omega}_{180}\right|$ rather than $\hat{\omega}_{180}$ is that $\left|\hat{\omega}_{180}\right|$ is a positive number while the estimator for $\omega_{180}$ may arrive at a negative solution. The block diagram of Fig. 4.1 also shows that a lowpass filtering is introduced in order to remove the effect of the oscillations in the estimate $\hat{\omega}_{\mathbf{1 8 0}}$.

\subsection{Adjustment of the perturbation signal}

The perturbation signal $(\delta y(t))$ gives a response in the system deviation which comes in addition to the natural system deviation caused by process disturbances, and should therefore be kept as small as possible. On the other hand, the "signal to noise ratio' i.e., the ratio of the variance of the perturbation signal to the variance of the disturbance, both measured at the system deviation, should have a reasonable value in order for the variance of the parameter estimates to be acceptable. At any rate it is of interest to adjust the amplitude of the perturbation signal so that the 'signal to noise ratio' is kept under control. An estimate of the disturbance response can be derived from the block diagram of Fig. 3.4 as $\delta e$. This estimate is proper when $\hat{\omega}_{180}=\omega_{180}=$ constant (see also section 3.2, (3.7)). In Fig. 4.2 a block diagram is shown based upon Fig. 3.4 for the automatic adjustment of the perturbation amplitude $\Delta y$. It determines the variance of the estimated perturbation response

$$
\overline{(\hat{e}(t))^{2}}
$$

and the variance of the estimated disturbance response

$$
\overline{(\delta e(t))^{2}}
$$

by establishing the relationship

$$
\eta(\delta e(t))^{2}-(\hat{e}(t))^{2}=T_{\Delta y}(\Delta \dot{y})
$$

where

$\eta$ : prescribed signal to noise-ratio 


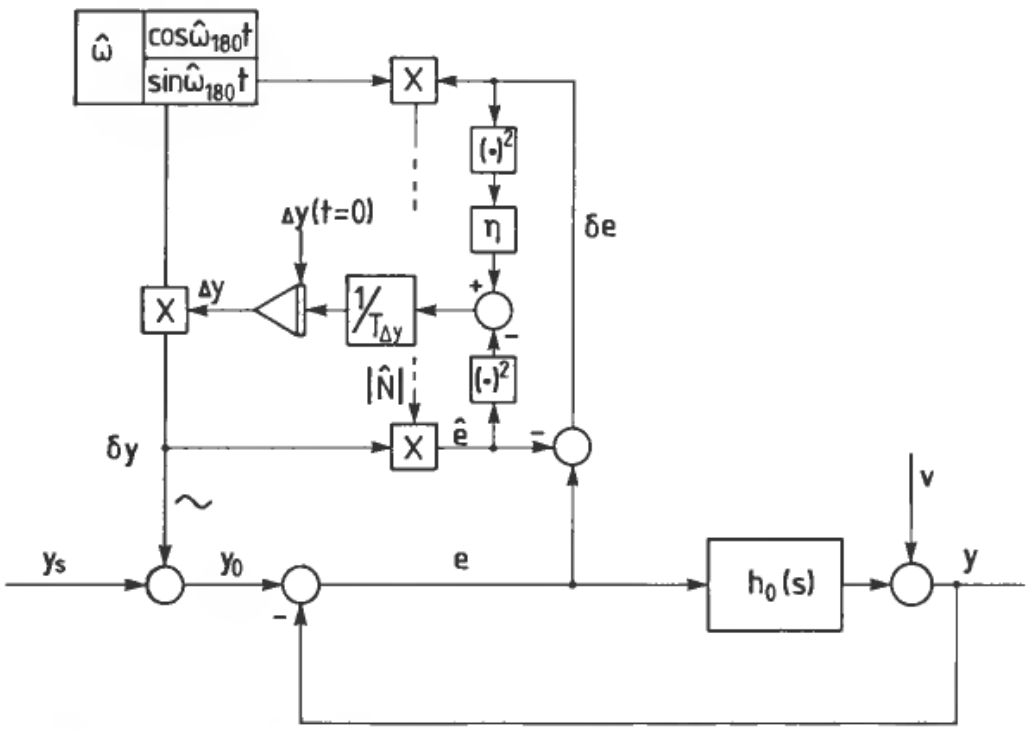

Figure 4.2. Scheme for adjusting the amplitude of the perturbation signal.

If the integration of (4.4) is made slowly enough, the perturbation amplitude $(\Delta y)$ will be adjusted until the steady state determined by

$$
\eta \overline{(\delta e(t))^{2}}=\overline{(\hat{e}(t))^{2}}
$$

is reached.

\section{Verification of the theory by experiments}

The estimator and the adaptive controller described in the previous sections have been tested on a laboratory process consisting of cascaded water vessels. It is sought to keep the water level $x$ in the last vessel in the sequence, close to a reference. See Fig. 5.1, where

$q$ : water flow rate into the cascaded vessels

$x$ : water level in the last vessel

$p$ : valve position for the valve at the water outflow from the system

$u$ and $y$ have been mapped linearly into the range $(0 \%, 100 \%)$. Linearization of the system above yields the following model:

$$
y=\frac{K}{\left(1+T_{1} s\right)\left(1+T_{2} s\right)\left(1+T_{3} s\right)} u
$$

With $y_{s}=50 \%$, the time constants of the system are approximately $T_{1}=T_{3} \approx 40 \mathrm{~s}$ and $T_{2} \approx 10 \mathrm{~s}$. In the first phase of the experiment, only the estimator was tested. The following controller parameters were used, see (4.1): $K_{p}=1 \cdot 5, T_{i}=100 \cdot 0, T_{d}=$ $0 \cdot 0$. A set of experimental runs was carried out under the conditions which are summarized in Table 1, where the initial value of $|\hat{N}|=2.0$ and the amplitude of the perturbation signal $\Delta y=1 \%$. Note that $p=$ 'partly closed' means that this valve is open and becomes partly closed during the run. 


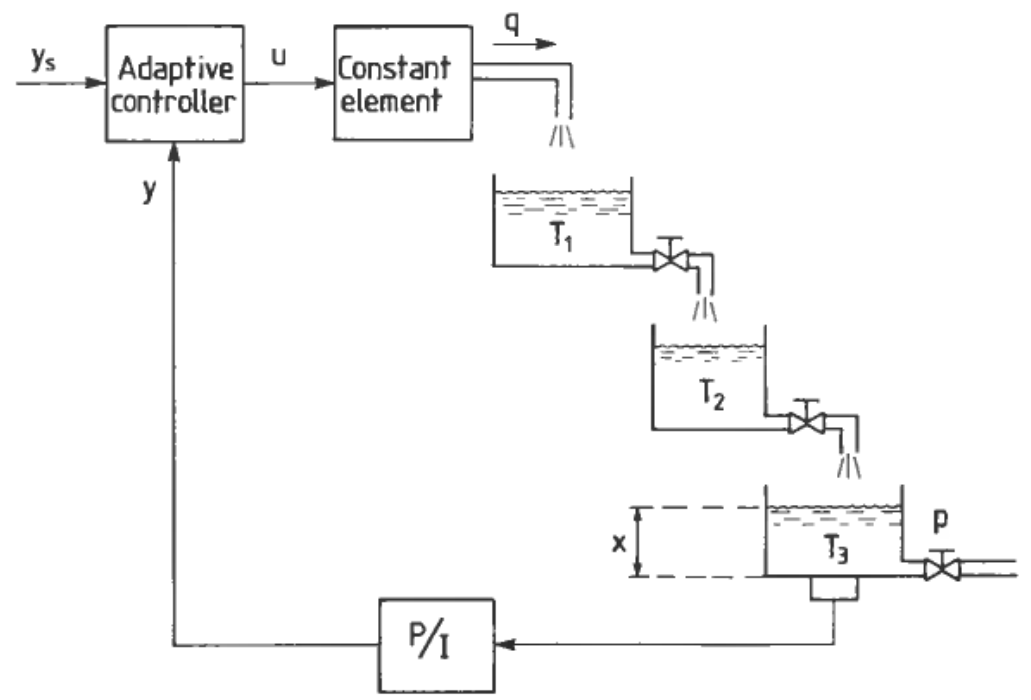

Figure 5.1. Basic experimental set-up.

\begin{tabular}{ccccccc}
\hline Run & $\begin{array}{c}\text { Initial value } \\
\text { of } \omega_{\mathbf{1 8 0}}\end{array}$ & $\alpha$ & $\beta$ & $y_{s}$ & $p$ & $\begin{array}{c}\text { Result in } \\
\text { figure }\end{array}$ \\
\hline 1 & 0.005 & 0.0005 & 0.05 & $50 \%$ & Open & $\left\{\begin{array}{l}5.2(a)-(b) \\
5.3\end{array}\right.$ \\
2 & 0.1 & 0.001 & 0.05 & $50 \%$ & Open & 5.2 \\
3 & 0.001 & $3.5 \mathrm{E}-4$ & 0.05 & $50 \%$ & Open & 5.3 \\
4 & $\begin{array}{c}\text { stationary } \\
\text { value }\end{array}$ & 0.0005 & 0.05 & $50 \%$ & $\begin{array}{c}\text { Partly } \\
\text { closed }\end{array}$ & 5.4 \\
\hline
\end{tabular}

Table 1. Conditions for the experiments carried out on the Estimator.

Figure 5.2 confirms the developed theory on the domain of attraction, see section 3.1. Since this process contains negligible amounts of time lag, we cannot expect to detect any $\omega_{H}$. In this figure, the estimate of $\omega_{180}$ is seen to oscillate around $\omega_{180}^{\circ} \approx 0.06$. At this frequency $|N(j \omega)| \approx 1 \cdot 7$. According to (3.6), the amplitude is (run no. 1)

$$
\Delta \omega \approx \frac{0.5 \times 10^{-3}}{4.0 \times 0.06} \times 1.7=3.510^{-3}
$$

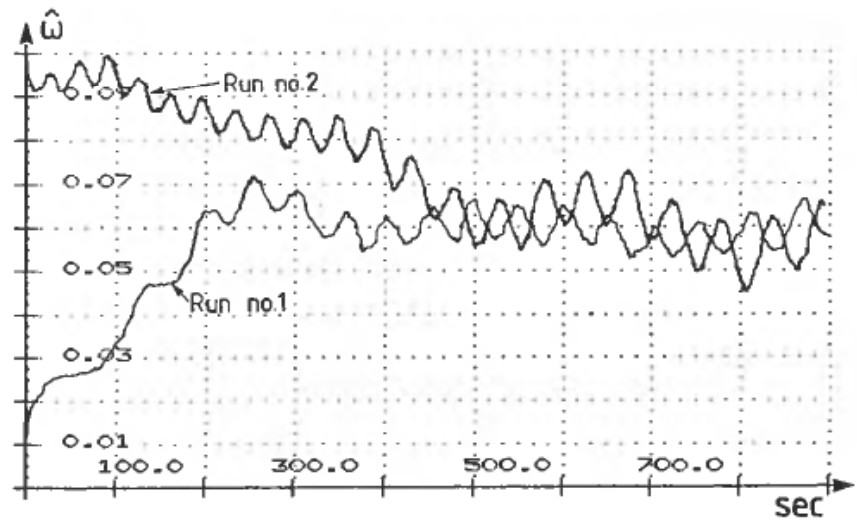

Figure 5.2(a). Estimate of $\omega_{180}$ using two different initial values. 


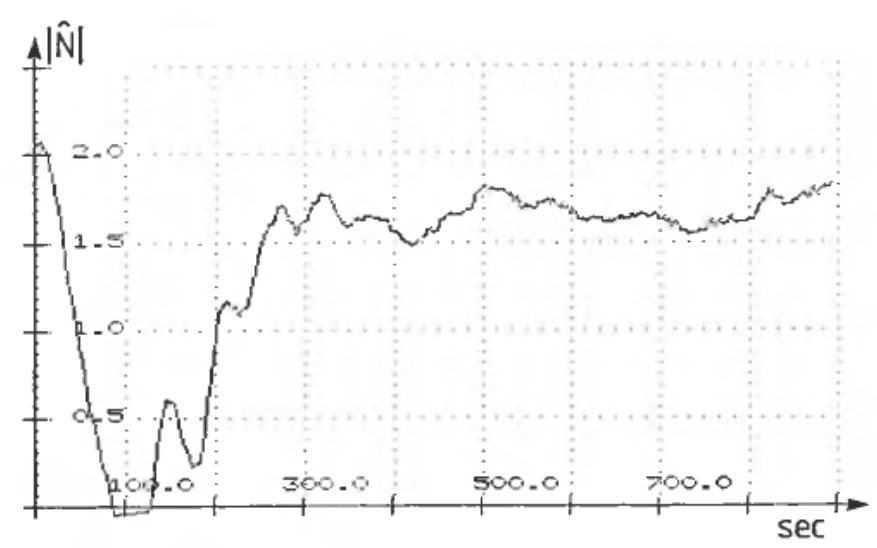

Figure 5.2(b). Estimate of $\left|N\left(j \omega_{180}\right)\right|$, run no. 1.

This is in good agreement with the results in Fig. 5.2. Figure 5.3 shows that the rate of convergence of $\hat{\omega}_{180}$ depends on the gain of the estimation loop, see (3.4).

When the valve at the outflow of the water from the system suddenly becomes partly closed during the run, the time constant of this vessel will suddenly increase. The time constants of the two other vessels will, however, decrease due to the lower steady states of their water levels. In addition to these effects, the gain of the process will change. The total effect of these parameter changes on the estimate of $\omega_{180}$, is shown in Figure 5.4.

In the last phase of the experiment, the full adaptive controller was tested. When there is a significant change in system parameters, large transient deviations may occur in $e$ and $\delta e$. As $e$ and $\delta e$ are inputs to the updating of $|\hat{N}|$ and $\hat{\omega}_{180}$, there should be no updating of these estimates when $e$ and $\delta e$ exceed some predetermined limits (run no. 5). The following initial values for the controller scheme were used: $K_{p}=1 \cdot 5, T_{i}=100 \cdot 0, \hat{\omega}_{180}=0.001,|\hat{N}|=2 \cdot 0$. In addition, the following constants were used: $\alpha=0.510^{-3}, \beta=0.02$ (run no. 5), $\beta=0.005$ (run no. 6 ), $\gamma=0.5, \delta=0.4$, $\eta=1 \cdot 0, \tau_{K}=10^{3}, \tau_{T}=10^{3},|N|_{0}=2 \cdot 0$. When the steady state was reached for the variables $\left(\hat{\omega}_{180},|\hat{N}|, K_{p}, T_{i}\right)$, experiments were performed as summarized in Table 2.

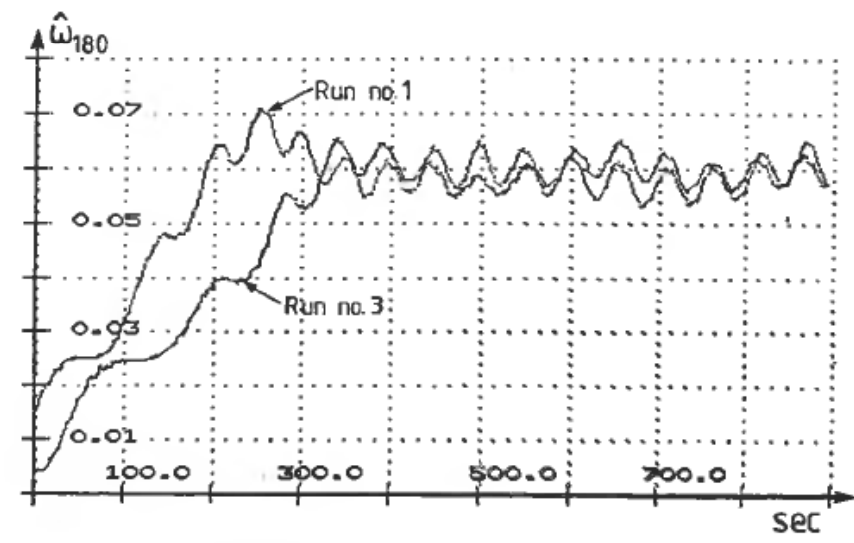

Figure 5.3. Estimate of $\omega_{\mathbf{1 8 0}}$ using two different loop gains. 


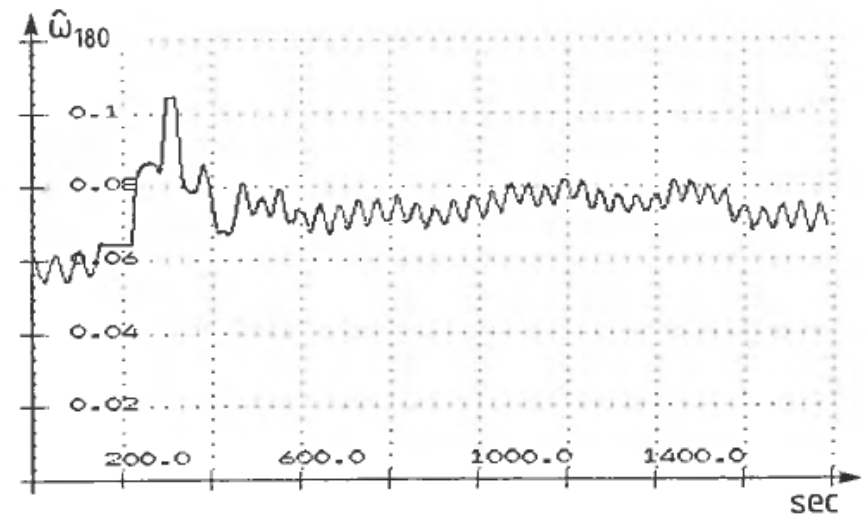

Figure 5.4. Estimate of $\omega_{180}$, run no. 4 .

\begin{tabular}{ccccc}
\hline $\begin{array}{c}\text { run } \\
\text { no. }\end{array}$ & $y_{s}$ & $p$ & $\Delta y$ & $\begin{array}{c}\text { Result in } \\
\text { figure }\end{array}$ \\
\hline 5 & $50 \%$ & $\begin{array}{l}\text { Partly } \\
\text { closed }\end{array}$ & $1 \cdot 0$ & $5 \cdot 5(\mathrm{a})-(\mathrm{d})$ \\
6 & $50 \%$ & Open & $\begin{array}{l}\text { Adapted with } \\
1.0 \text { as initial } \\
\text { value }\end{array}$ & $5.6(\mathrm{a})-(\mathrm{i})$ \\
\hline
\end{tabular}

Table 2. Conditions for the experiments on the adaptive controller.

Figure 5.5 confirms that the adaptive controller works with sudden changes in the parameters of the process. Figure 5.6 shows that the adaptation of $\Delta y$ works satisfactorily. Note how the size of $e$ approaches that of $\delta e$, and how the sinusoidal variation in $u$ approaches white noise.

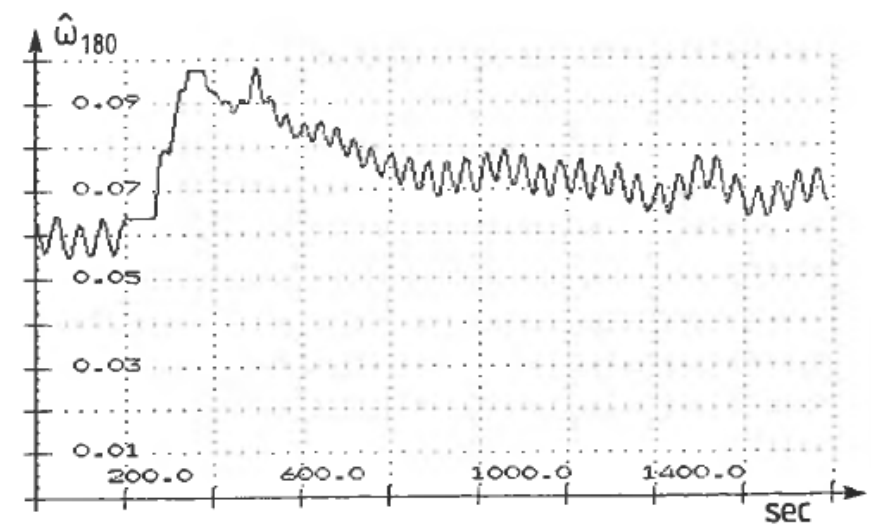

Figure 5.5(a). Estimate of $\omega_{180}$, run no. 5 . 


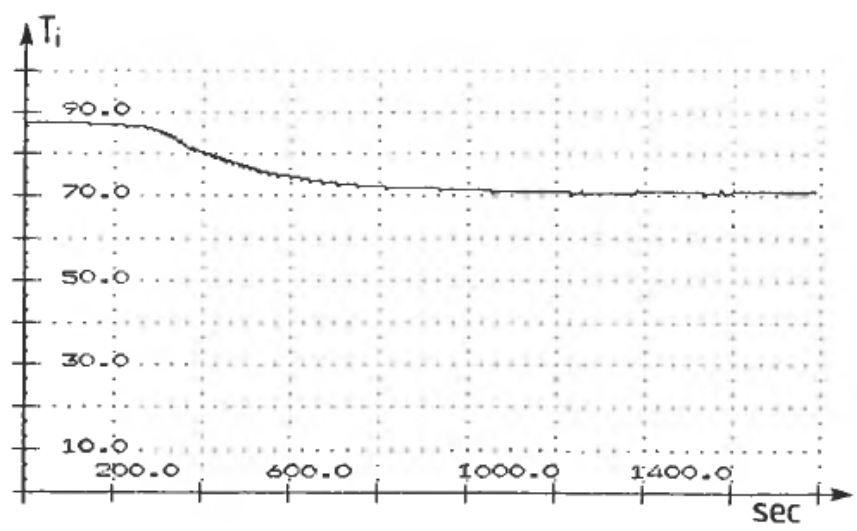

Figure 5.5(b). Integral time in the controller, run no. 5.

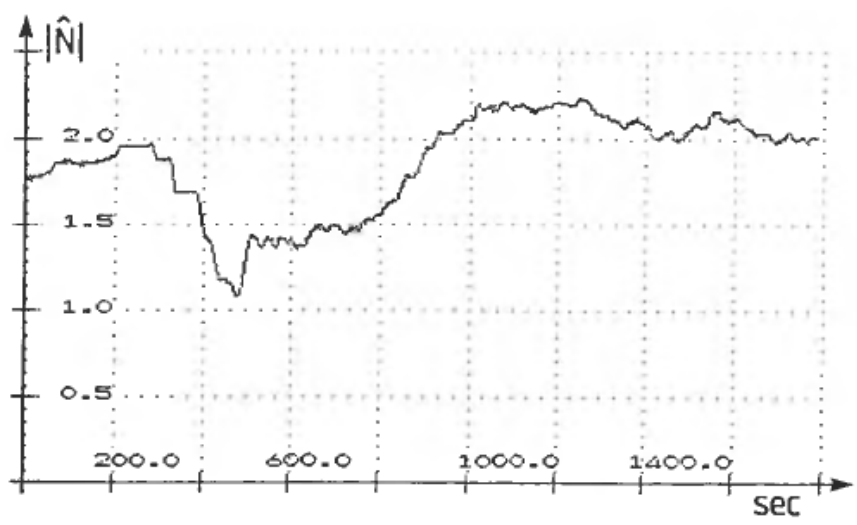

Figure 5.5(c). Estimate of $\left|N\left(j \omega_{180}\right)\right|$, run no. 5 .

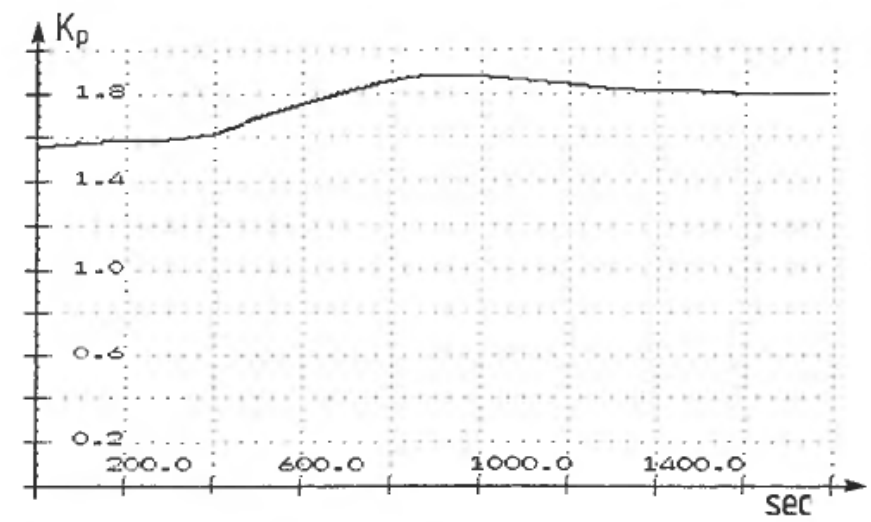

Figure 5.5(d). Proportional gain in the controller, run. no. 5. 


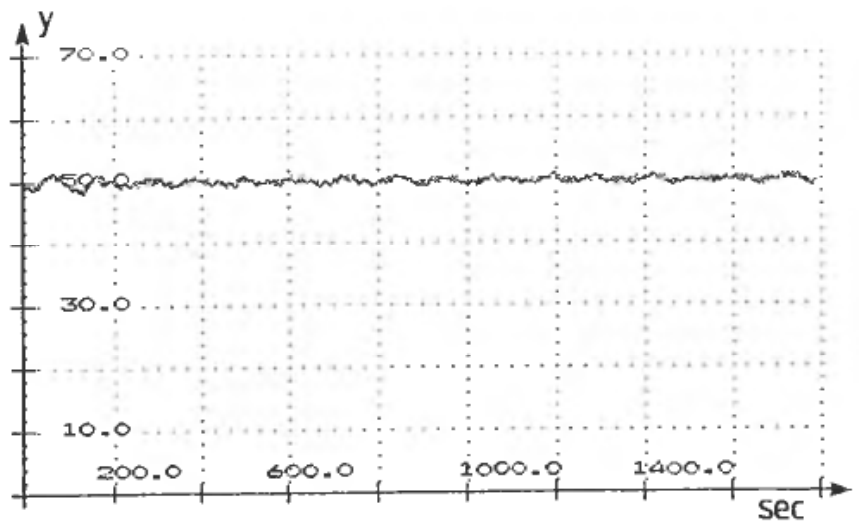

Figure 5.6(a). Time response of the measurement $y$, run no. 6.

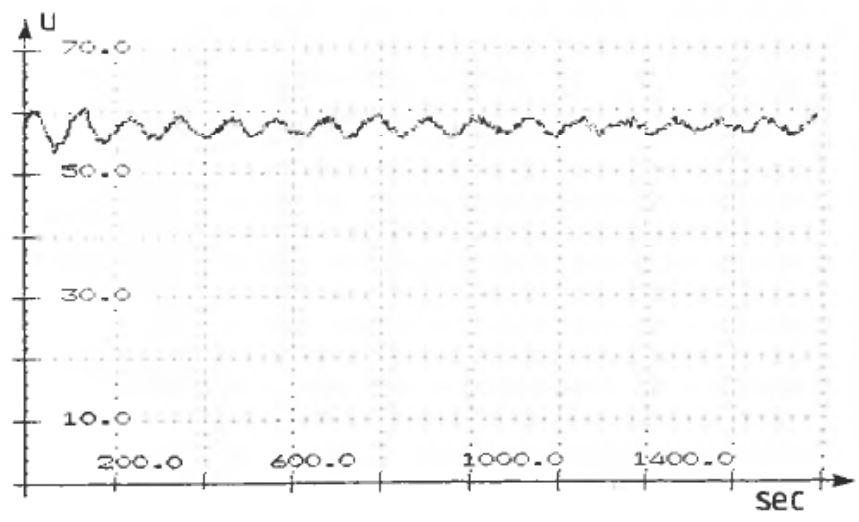

Figure 5.6(b). Time response of the control variable $u$, run no. 6 .

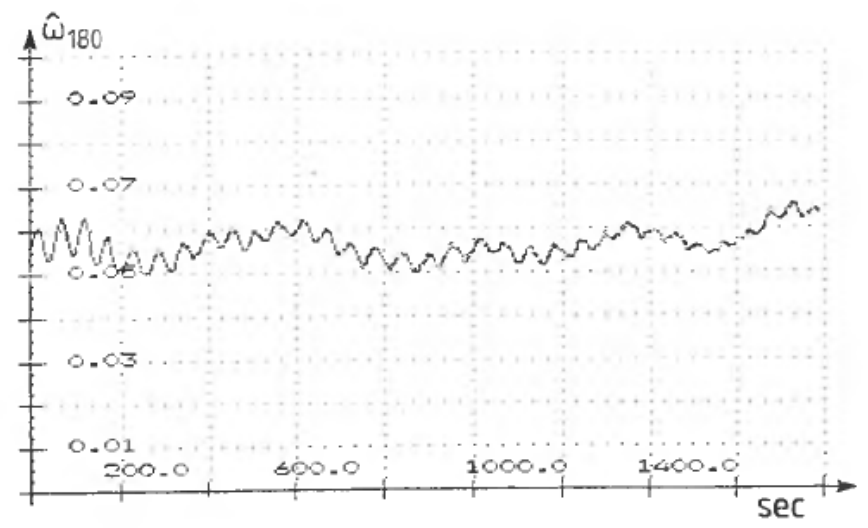

Figure 5.6(c). Estimate of $\omega_{180}$, run no. 6 . 


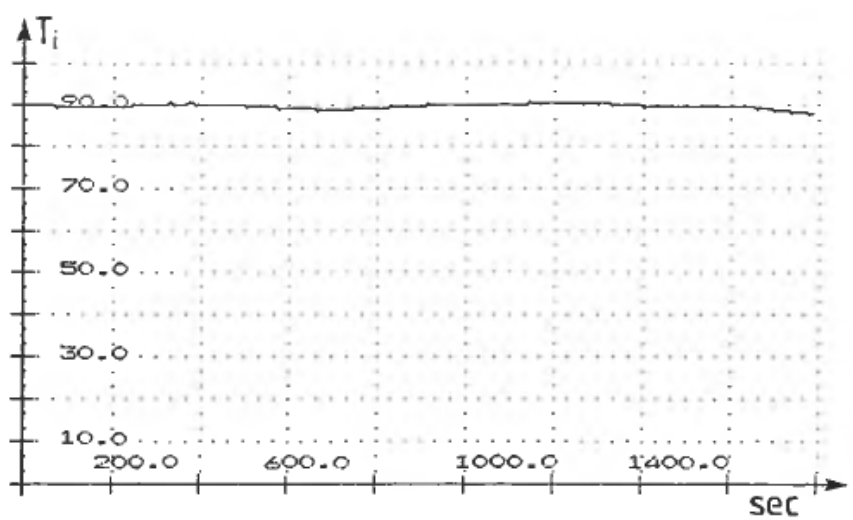

Figure 5.6(d). Integral time in the controller, run no. 6 .

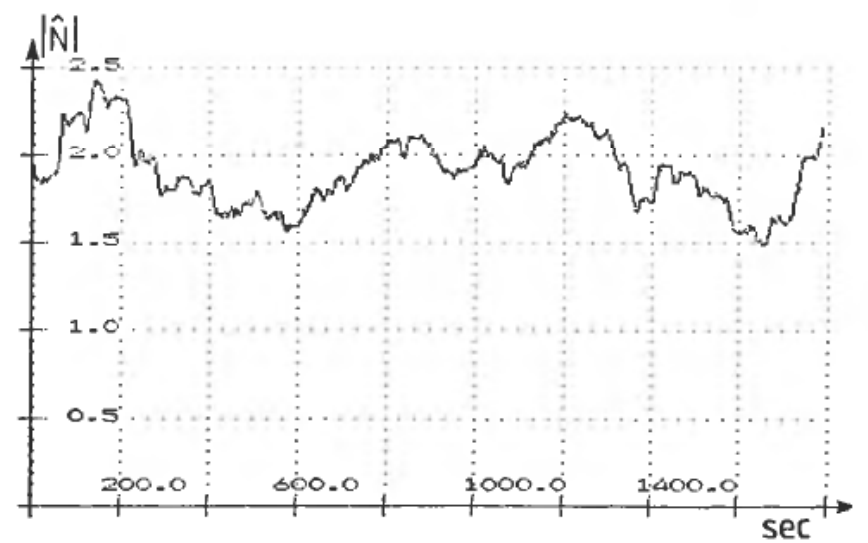

Figure 5.6(e). Estimate of $\left|N\left(j \omega_{180}\right)\right|$, run no. 6 .

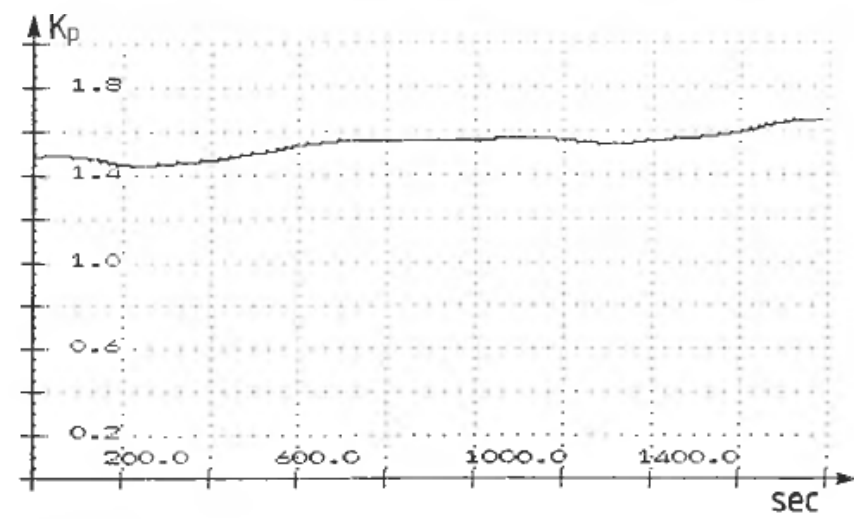

Figure 5.6(f). Proportional gain in the controller, run no. 6. 


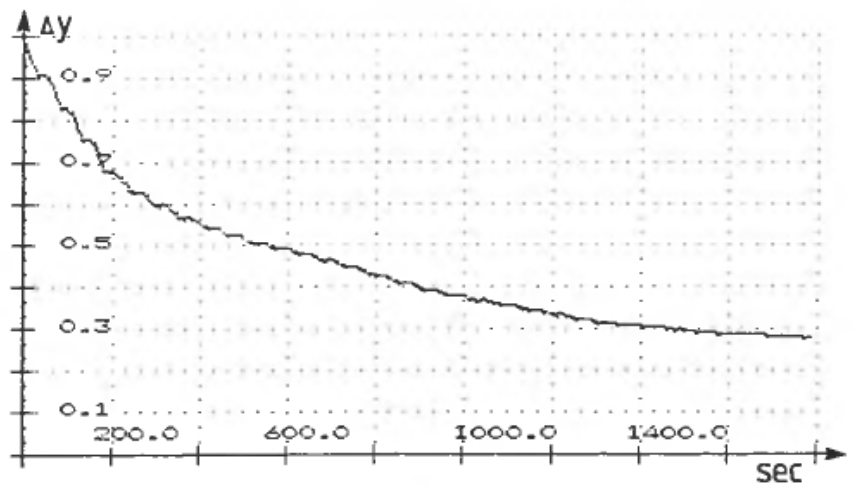

Figure 5.6 $(g)$. Amplitude of the perturbation signal, run no. 6 .

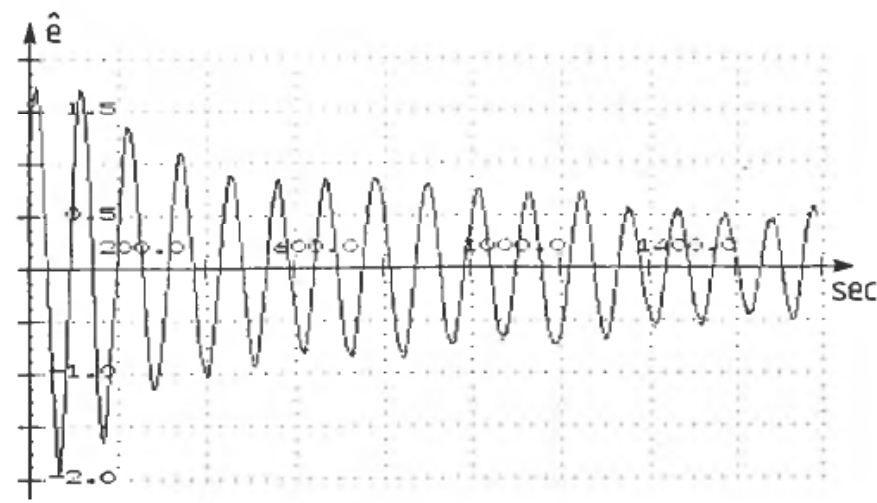

Figure 5.6(h). Estimate of system deviation caused by the perturbation signal, run on. 6 .

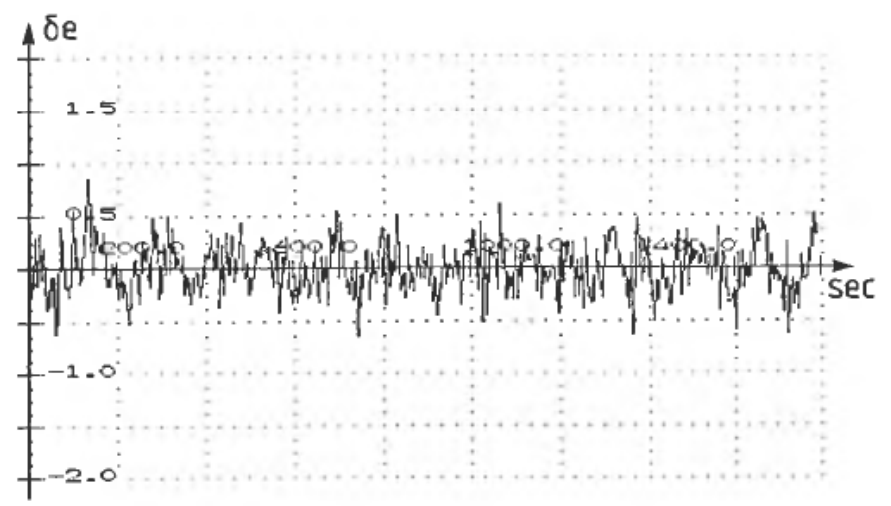

Figure 5.6(i). Estimate of system deviation caused by the process disturbance, run no. 6. 


\section{Conclusion}

A new adaptive controller has been developed based upon on-line estimation of the -180 degree phase shift frequency $\left(\omega_{180}\right)$ and the gain margin. These estimates are used to update the parameters in a control algorithm, exemplified by a PID controller. The estimator incorporates the tuning of the perturbation amplitude in order to make the excitation sufficient but not excessive. This tuning is important when controlling real processes where the size of the output disturbance is unknown. Laboratory experiments verify the theory, and the behaviour of the controller was satisfactory.

It is apparent that the variance of the estimate of $\omega_{180}$ is somewhat sensitive to disturbance frequency components in the neighbourhood of $\omega_{180}$. This means that care should be taken if the disturbance is strongly dominated by components in this frequency domain.

In the algorithm presented in this paper, constants such as the gains of the estimation loops, initial values, etc., are assumed to be known. Most of these quantities can be determined through simple automatic experiments on the process, thus minimizing the efforts of the user.

\section{ACKNOWLEDGMENTS}

We would like to thank Eivind Johan Lund and Øystein Strømsnes who implemented the algorithm on a digital computer and performed the experiments as a part of a student project at Division of Engineering Cybernetics.

\section{REFERENCES}

Åström, K. J., BoRIsSON, U., LJUNG, L., and WitTENMARK, B. (1977). Theory and applications of self-tuning regulators. Automatica, 13, 457-476.

Feldbaum, A. A. (1960). Dual Control Theory I-II. Automation and Remote Control (Transl. from Russian), 21, 9 and 11 (Instrument Society of America).

LJUNG, L. and SöDERSTRÖM, T. (1985): Theory and practice of recursive identification. 2nd ed. (The MIT Press; Cambridge, Massachusetts). 